\title{
La traduction comme création: Entretien avec Gao Xingjian
}

\author{
Mingxing Wang \\ University of Alberta
}

Récipiendaire du prix Nobel de littérature, Gao Xingjian est notamment l'auteur de La Montagne de l'âme et de plusieurs pièces de théâtre. Cela dit, on ignore souvent qu'il a également signé plusieurs traductions. Cet entretien réalisé avec Gao Xingjian le 10 septembre 2015 à l'occasion du $5^{\mathrm{e}}$ Congrès des études asiatiques et pacifiques qui s'est tenu à L'Institut National des Langues et Civilisations Orientales (INALCO) à Paris, est l'occasion d'apporter un éclairage sur son travail de traducteur. L'entretien a été réalisé en chinois, puis traduit en anglais et en français.

Wang : Merci de prendre le temps de parler avec moi de vos traductions. À mon avis, votre carrière littéraire est liée à la traduction, qu'en pensezvous?

Gao : C'est vrai simplement dans une certaine mesure.

Wang: Parlons un peu du début de votre carrière comme traducteur. Quand vous étiez étudiant au département d'études françaises de l'Institut des Langues Étrangères de Beijing au début des années 1960, aviez-vous suivi une formation en traduction et pratiqué la traduction?

Gao: À cette époque, mon programme n'offrait pas de formation en traduction. Je n'ai pas fait de traduction non plus. Mais on avait une grande liberté dans nos études au sein du département.

Wang: Aussi loin que je me souvienne, dans les années 1970, vous travailliez comme traducteur vers le français pour la librairie internationale de Chine, puis comme chef d'équipe pour la section française du journal La Chine en construction. Que traduisiez-vous alors?

Gao : En fait, je ne traduisais pas beaucoup...

Wang : Vraiment? 
Gao : En effet.

Wang : Alors, quand avez-vous commencé à traduire?

Gao : J'ai seulement commencé vers 1976, à la mort de Mao.

Wang: Que traduisiez-vous? Aviez-vous une préférence particulière pour un type de texte?

Gao: Je traduisais beaucoup de textes et je les proposais à la publication seulement quand mes amis me demandaient de contribuer à leur journal littéraire. À cette époque, je ne choisissais que les textes qui répondaient à leurs besoins.

Wang: En parlant de traduction dans les années 1970, le grand poète Bei Dao dit dans un texte: «la traduction est une rébellion silencieuse ", c'est-à-dire que la traduction joue un rôle important en aidant à voir le monde d'un point de vue complètement différent. Pensez-vous que la traduction peut être considérée comme un acte de rébellion.

Gao : En ce qui le concerne, c'est peut-être vrai. Dans mon cas, le français m’a permis de connaître le monde extérieur. Je lisais beaucoup en français.

Wang: Quand vous avez choisi de traduire les pièces de Samuel Beckett et d'Eugène Ionesco, vous êtes-vous identifié à ces écrivains bilingues?

Gao : Non.

Wang: Au début des années 1980, vos nouvelles ont été traduites en français, n'est-ce pas?

Gao : Oui. Paul Poncet qui était professeur de langue et de littérature chinoises a signé les traductions françaises de mes nouvelles «Dans un parc», puis «Mère » dans les années 1980. Après sa mort, j’ai demandé au professeur Noël Dutrait de prendre la relève.

Wang : Quand le professeur Noël Dutrait a commencé à travailler sur Lingshan (La Montagne de l'âme) au début des années 1990, avez-vous collaboré avec lui? 
Gao: Non, je n'ai pas collaboré avec lui, mais j’ai été au courant de ce qu'il faisait.

Wang: En plus de la version française, votre roman Lingshan a été traduit en suédois et en anglais dans les années 1990. Votre traducteur suédois, Göran Malmqvist, a qualifié votre langue de " pure » alors que votre traductrice anglaise, Mabel Lee, a caractérisé votre style comme étant minimaliste. Êtes-vous d'accord avec ces opinions?

Gao : Minimaliste? Non. Mais il est vrai que ma langue est pure.

Wang: En ce qui concerne les versions bilingues de vos pièces écrites en France, Claire Conceison, la traductrice anglaise de Ballade nocture a dit que quand elle a traduit votre pièce en anglais, elle a dû prendre en compte vos autres pièces pour en faire sorte que sa traduction leur ressemble. Qu'en pensez-vous?

Gao : Cela dépend de la nature de mes pièces. Certaines sont comparables entre elles, d'autres pas.

Wang : À plusieurs occasions, vous avez dit que vous vouliez utiliser la littérature comme un moyen d'exprimer votre voix singulière d'écrivain. Cela dit, pour ce qui est de la traduction, votre voix singulière doit être exprimée dans une autre langue par un traducteur. Où se trouve votre voix? N'est-elle pas étouffée?

Gao : Il faut espérer qu'il y ait une meilleure solution!

Wang : Qu'en est-il de votre propre approche de la traduction? En 2013, vous avez donné une présentation sur la traduction au Heng Seng Management College à Hong Kong. Il me semble que c'est le modèle proposé par le célèbre traducteur chinois Lin Shu $(1852$ - 1924) qui a votre préférence. Avez-vous essayé de traduire à la manière d'Ezra Pound?

Gao : Oui. 